\title{
Measured Resection Versus Gap Balancing for Total Knee Arthroplasty
}

\author{
Matthew P. Abdel MD
}

\section{Introduction}

r he number of TKAs performed in the United States is expected to increase substantially in the next two decades [6]. Additionally, surgeons are performing TKAs in younger and more active patients. While many articles discuss the importance of radiographic alignment, soft-tissue balancing is essential to a successful TKA. There remains great controversy in how surgeons obtain

The author certifies that he or any members of his immediate family, have no funding or commercial associations (eg, consultancies, stock ownership, equity interest, patent/ licensing arrangements, etc) that might pose a conflict of interest in connection with the submitted article.

All ICMJE Conflict of Interest Forms for authors and Clinical Orthopaedics and Related Research editors and board members are on file with the publication and can be viewed on request.

The opinions expressed are those of the writers and do not reflect the opinion or policy of $C O R R^{\mathbb{R}}$ or the Association of Bone and Joint Surgeons ${ }^{\circledR}$.

M. P. Abdel MD ( $\varangle)$

Department of Orthopedic Surgery,

Mayo Clinic, 200 First Street SW,

Rochester, MN 55905, USA

e-mail: abdel.matthew@mayo.edu correct alignment and soft-tissue tensioning. Some surgeons utilize a measured resection technique, while other surgeons favor a gap-balancing technique.

As such, I have invited two internationally known orthopaedic surgeons to discuss these two approaches, both of which seek to achieve the same general goal - a well-aligned and well-balanced TKA. Bryan D. Springer MD is an attending joint replacement surgeon at the OrthoCarolina Hip and Knee Center in Charlotte, NC, USA, and a member of The Knee Society. Dr. Springer is the recipient of numerous Knee Society Awards, and has extensive experience with the gap-balancing technique. Sébastien Parratte $\mathrm{MD}, \mathrm{PhD}$ is an attending joint replacement surgeon and assistant professor of orthopedic surgery at the University Hospital of Marseille in Marseille, France. Dr. Parratte likewise has received numerous Knee Society and Hip Society Awards, and has considerable experience with the measured resection technique.

Matthew P. Abdel MD: What do you each see as the advantages of the gapbalancing or measured resection techniques, in terms of achieving ligament balance?
Bryan D. Springer MD: Instability following TKA remains one of the most common modes of early failure. The gap-balancing technique is based on the progressive release of ligaments to create equal and symmetric flexion and extension gaps. It is imperative that one understands what structures affect extension, flexion, and both in order to create proper balance.

One of the fundamental differences between the gap-balancing technique and measured resection technique lies in setting the rotation of the femoral component to achieve a symmetric flexion gap. Measured resection relies on arbitrary bony landmarks (surgical transepicondylar axis, posterior condylar axis, and anteroposterior (AP) axis of the femur) in order to set rotation. While bony landmarks may occasionally provide accurate rotation of the femoral component, one major disadvantage is the surgeon's inability to accurately and reproducibly find them intraoperatively. Research has suggested there is wide variability in femoral component rotation when using bony landmarks, which leads to asymmetry of the flexion space and condylar lift off (Fig. 1) in flexion [3].

Gap balancing relies on symmetric tension placed on the ligaments in 


\section{Clinical Faceoff}

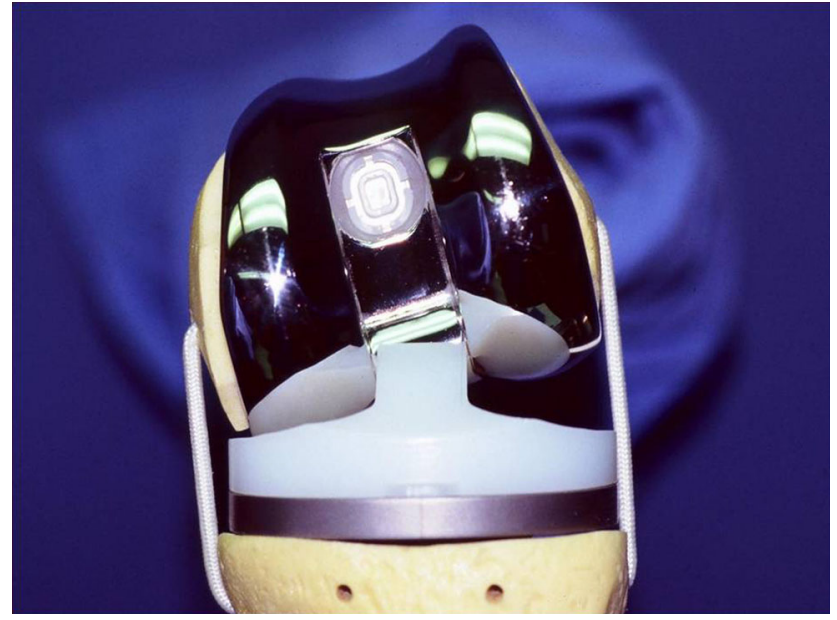

Fig. 1 This schematic depicts condylar lift off that occurs when there is asymmetry of the flexion space. Published courtesy of Bryan D. Springer MD..

flexion to set femoral component rotation. The femur is rotated to create an equal and symmetric flexion gap and the size of the flexion gap is adjusted to match the gap created in extension. Therefore, the rotation and AP placement of the femoral component are used to create flexion gap symmetry (as opposed to fixed bony landmarks). Multiple comparative studies have demonstrated better femoral component rotation, coronal stability, flexion gap stability, and patellar tracking compared to measured resection techniques $[3,7,9]$.

Sébastien Parratte MD, PhD: I agree with Dr. Springer that instability remains a significant concern with contemporary TKAs. Minimizing instability, particularly midflexion instability at $45^{\circ}$, is fundamental to achieving stability and a pain-free knee after arthroplasty. The gap-balancing technique may help surgeons obtain symmetric ligament tensions at $0^{\circ}$ and $90^{\circ}$ of flexion. However, in my opinion, it does not always allow for midflexion balancing, and therefore, the reason many surgeons still utilize a measured resection technique. This occurs with the gap-balancing technique for several reasons. Foremost, the tibial cut, which can be considered as a platform, is utilized to create the flexion space at $90^{\circ}$. Consequently, the tibial cut needs to be absolutely perfect as any varus or valgus malalignment may lead to femoral malrotation. Additionally, in the gapbalancing technique, it is difficult to accurately control the distraction forces.
For instance, the lateral compartment gap tends to increase greater than the medial compartment gap as the joint gap distraction force increases [10]. This can lead to femoral malrotation. Finally, having equal tensions of the ligament at $0^{\circ}$ and $90^{\circ}$ is not enough to prevent midflexion instability. In fact, a recent study demonstrated that a significant proportion $(36 \%)$ of TKAs showed midflexion laxity even when rectangular extension and flexion gaps were achieved at $0^{\circ}$ and $90^{\circ}$, respectively [12].

Dr. Abdel: What role does the subluxed extensor mechanism play in balancing the knee with a gap-balancing technique or measured resection technique? Dr. Springer: Whether subluxed or reduced, the role of the extensor mechanism when determining femoral component rotation and gap balancing in flexion remains controversial. When tensing the flexion gap to set rotation, how does the extensor mechanism affect the size and tension placed on the gap? Many tension devices allow for gap tensioning in flexion to be done with the extensor mechanism reduced, while others require the extensor mechanism to be subluxed.

We have performed some preliminary analyses in our laboratory using a mechanical pressure sensor device looking at several parameters in gap balancing. One parameter was the difference in the gap size and tension 


\section{Clinical Faceoff}

with the extensor mechanism reduced or subluxed. Interestingly, we were unable to show any substantial difference in the gap size or tension on the lateral side of the knee with the extensor mechanism reduced or subluxed when using a gap-balancing technique.

A measured resection technique, however, may lead to a larger lateral flexion gap. The lateral side of the knee in flexion is inherently more lax and elastic than the medial side of the knee. This is particularly true for a varus pattern of arthritis, with a contracted medial side in flexion. Since measured resection relies on fixed bony landmarks to set rotation, the lateral laxity is not taken into consideration and leads to asymmetry in rotation and a trapezoidal flexion gap (a varus flexion gap). Gap balancing compensates for this lateral laxity by adjusting rotation under tension to create an equal and symmetric flexion gap.

Dr. Parratte: I completely agree with Dr. Springer that the role of the subluxed extensor mechanism during TKA remains controversial. In the gap-balancing technique, most of the tensioners are used with the patella subluxed. As such, the gap may not be properly sized and balanced, which may lead to excessive internal rotation of the femur. For measured resection, however, the position of the patella during the procedure does not influence femoral rotation. In fact, rotation is determined based upon the available bony landmarks (posterior condyles, Whiteside's line, and surgical transepicondylar axis). The combination of bony landmarks used in measured resection seems to better optimize the position of the femoral component [5]. In addition, in a recent study [11] evaluating the rotation of the femoral component using a measured resection technique versus patient-specific instrumentation, we were able to show good agreement utilizing both techniques.

Dr. Abdel: What are the differences between the gap-balancing and measured resection techniques in terms of the soft-tissue releases needed to achieve ligament balance, and to what degree do they differ in terms of changes to the joint line and/or femoral component rotation?

Dr. Springer: I believe that most surgeons use a combination of techniques to achieve the same goals. For example, I typically make a standard measured resection cut off my distal femur to maintain femoral joint line position. As mentioned above, the fundamental difference between gap balancing and measured resection lies in setting the rotation of the femoral component. There are, however, several other differences in the surgical technique.
With gap balancing, the extension gap is created by bony resection of the distal femur and proximal tibia. Equal medial and lateral gaps are created in extension by a stepwise release of tight structures on the concave side. For example, in a varus knee, tight structures in extension are the deep medial collateral ligament (MCL) and posteromedial corner of the knee. Releasing these structures will affect the extension space more than the flexion space.

What one must understand is that any releases that are done in extension, on either the medial or lateral side of the knee, can have an unpredictable effect on the flexion space [4]. Gap balancing accommodates for this by allowing the flexion space to be set based on ligament tension and accommodates for releases that have already been made in extension, rather than fixed bony landmarks. With a measured resection technique, releases are made with trial component in place after all bony resections have been made and femoral component rotation is set off of arbitrary bony landmarks. Releasing the tight medial side of the knee in extension for example, can then lead to the creation of laxity and asymmetry in flexion.

Dr. Parratte: As mentioned by Dr. Springer, the creation of the extension gap is common to both techniques and can be considered as a measured 


\section{Clinical Faceoff}

resection technique. Importantly, the joint line is primarily determined at this step. Multiple studies [1, 7] have shown that with the gap-balancing technique, the joint line is elevated in relation to the measured resection technique to create symmetrical gaps. While the amount of joint elevation varies, and the clinical consequences are unknown, those who use a gapbalancing technique should be cognizant of the elevation.

It is also important to point out again that the tibial cut, in terms of depth of resection and alignment (both in the coronal and sagittal planes), is absolutely essential. In the gap-balancing technique, any deviation will result in femoral component malrotation. As such, regardless of technique, I always utilize trial components to determine stability in the both the coronal and sagittal planes throughout the entire arc of motion.

Dr. Abdel: To what degree do other factors such as implant selection (cruciate-retaining versus cruciatesubstituting) or preoperative coronal alignment (varus or valgus) influence one's choice of approach between measured resection and gap balancing?

Dr. Parratte: Surgeon experience and familiarity with a particular implant likely plays the greatest role, regardless if a measured resection technique or gap-balancing technique is utilized. There are expert cruciate-retaining surgeons using the measured resection technique and expert cruciate-substituting surgeons using the gap-balancing technique, and vice-versa. Today, there are likely more measured resection and cruciate-substituting users in the North America. In Europe, there is great variability among and within countries. As a user of cruciate-substituting implants, I feel that the best way to manage the flexion space void of the posterior cruciate ligament (PCL) is to utilize a hybrid technique, as I will describe in a moment.

In regards to the effects of preoperative coronal alignment on technique choice, the main question is if the deformity is reducible or not. I feel strongly about obtaining stress-view radiographs in such patients. If the deformity is reducible, then both techniques can be used. If the deformity is not reducible, ligament releases may be required. In these instances of fixed deformities, the gap-balancing technique may be limited. For example, in a patient with a severe, fixed valgus deformity of the knee, the MCL may be deficient. As such, a gapbalancing technique would not allow for appropriate ligament balancing.

Dr. Springer: I agree with Dr. Parratte in that surgeon experience and familiarity with a particular implant play the greatest role, regardless of technique. I am a user of cruciate-substituting implants as well, and feel, again, in my hands the combination of a gap-balancing technique and a cruciatesubstituting implant allows for the most reproducible results. Excellent results can certainly be achieved with either surgical technique or either implant. However, one must understand the difference that the PCL plays in the knee when intact or removed. When the PCL is removed, the flexion gap immediately opens up (about $2 \mathrm{~mm}$ ). Therefore, one must compensate for this by taking some additional initial bone off the distal femur to open up the extension gap. For instance, the distal femoral cut in a cruciate-retaining TKA may be $7 \mathrm{~mm}$ to $8 \mathrm{~mm}$ and $9 \mathrm{~mm}$ to $10 \mathrm{~mm}$ in a cruciate-substituting knee (assuming no flexion contracture). Additionally, releases on the medial side of the knee will have less effect when the PCL is intact. When the PCL is removed for a cruciate-substituting knee, the releases on the medial side of the knee will have more of an effect in flexion.

I also strongly agree that preoperative determination of correction of deformity is paramount in every knee. While I do not routinely obtain stress views, I insist on examining every knee under anesthesia prior to prepping and draping, to determine the amount of correction of the deformity. This, in turn, determines how much 


\section{Clinical Faceoff}

release I will do during the initial part of the procedure. We are always trying to avoid over releasing ligaments and playing catch up. Determining the ligamentous "personality" of the knee is important.

Dr. Abdel: What research on patientreported outcome measures (PROMs) or survivorship might guide a surgeon in deciding between the measured resection and gap-balancing techniques?

Dr. Parratte: If I had to choose between a measured resection technique or gap-balancing technique based upon PROMs, it would be difficult to make a decision based on the current literature. There are a limited number of series comparing PROMs between the two techniques and in these series, no difference has been observed [2, 8]. Regarding survivorship, long-term followup studies have reported good survival with both techniques. However, it is intriguing to note that even though this debate started in the 1970s, no study has shown a definitive survivorship benefit between the two techniques. In reality, most of the literature compares femoral component rotation and kinematics. For both techniques, the main limitation is trying to link differences in PROMs to the approach utilized, when there are several factors that may influence the PROMs. Moreover, we are still unable to quantify intraoperative ligament tensioning and balance, as well as patellar tracking. The second limitation involves knowing what the targeted femoral component rotation should be in each patient. How many degrees of external rotation should be given to the femoral implant: $0^{\circ}, 3^{\circ}$, or more? Should we set the same rotation for everybody? Can we truly establish the rotation based on the ligament tension without having an objective idea of what this tension should be? Finally, it is important to note that the knee is a dynamic joint, yet we make relatively static decisions intraoperatively regarding ligament balancing and component alignment.

Dr. Springer: I agree with Dr. Parratte. Based on the current literature, I think it would be difficult to make a determination as to which technique, gap balancing or measured resection, has better PROMs. One reason may be that our current scoring systems are not sensitive enough to detect subtle differences in patient outcomes. Therefore, most patients are generally grouped together.

Rather than trying to decide which technique has overall better outcomes, it is important to determine in an individual surgeon's hands, which technique provides the most reliable and reproducible results. Hopefully, we all agree that the goals of TKA are the same, regardless of the technique that is used. A well-aligned, well-balanced, and stable knee is associated with improvements in patient function and satisfaction. Which technique best achieves this is controversial. In my hands, a gap-balancing technique most reliably and reproducibly allows me to achieve the above-mentioned goals. As with most surgery, if it is done well, the goals and outcomes can be achieved, regardless of technique.

Dr. Abdel: What alternative techniques are worth talking about (perhaps such as a hybrid technique) for TKA apart from strict measured resection or gap balancing, and how do they compare in your mind against these 'classic' approaches?

Dr. Parratte: This question is the key point. We should keep in mind that patients desire a TKA that is mobile, pain free, and stable in the long-term. While this may seem obvious in 2014, the fact remains that almost $20 \%$ of the patients after TKA are not happy due to a host of reasons, including limited ROM, continued pain, and/or instability. Moreover, this occurs with both measured resection and gap-balancing techniques, indicating that further investigation of a hybrid technique is required. At the current time, I utilize all bony landmarks and soft-tissue clues to maximize appropriate alignment and soft-tissue balancing. In 2014, I utilize a hybrid technique that 


\section{Clinical Faceoff}

starts with measured resection of the proximal tibia and distal femur, but then focuses on three gaps - the extension gap, the flexion gap, and the patellofemoral gap. I initially set my femoral rotation using a standard jig and all available bony landmarks (posterior condyles, Whiteside's line, and surgical transepicondylar axis). Then, I place my femoral cutting block on and check my flexion space in regards to ligament tensioning and medial-lateral stability (Fig. 2). I adjust my component rotation based upon this analysis. I complete this with the extensor mechanism subluxed due to limitations in contemporary instrumentation. In the future, it will be essential to assess the flexion space and patellofemoral gap with the extensor mechanism reduced.

The concept of a hybrid technique is ideal because TKA is both a soft-tissue and bony procedure. Combining the two techniques, with established intraoperative checks-and-balances between the two techniques, allows for minimization of inappropriate ligament releases and component malalignment. The risk of using a strict gap-balancing technique is that this is rather subjective, and can introduce significant bias not only between surgeons, but also within a surgeon's practice. Also, as noted above, almost all gap-balancing tensioners are used with the patella subluxed, which influences the

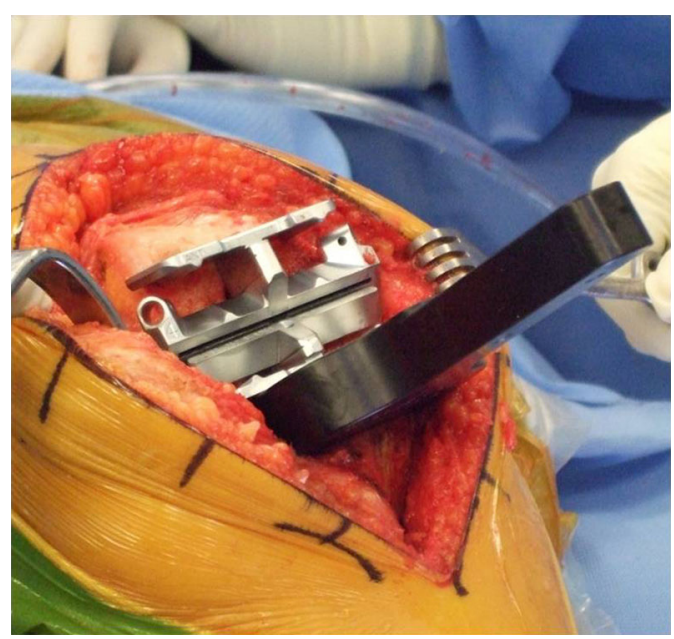

Fig. 2 The hybrid technique involves placing an appropriately sized spacer block underneath the femoral cutting block when the knee is in $90^{\circ}$ of flexion. At this point, rotation can be adjusted to ensure a symmetrical flexion space. Published courtesy of Bryan D. Springer MD.

ligament tension between the medial and lateral sides. This may lead to femoral component malrotation.

Dr. Springer: I believe that most surgeons, myself included, who are gap balancers or measured resectors, probably are not purists. I suspect most of us use a combination of gap-balancing and measured resection techniques when performing a TKA. For example, my distal femoral cut and proximal tibial cut are measured resection cuts, allowing for maintenance of joint line position. My femoral component rotation and flexion gap balancing are done with a gap-balancing technique. This allows the strengths of each technique to be combined, while minimizing some of the concerns with each technique such as elevation of joint line with gap-balancing and malrotation of the femoral component with measured resection.

I often say that the way I currently perform a TKA may be nothing like how I perform a TKA in 10 years, and is certainly not how I performed one 10 years ago. I strongly believe that a better understanding of individual anatomy, alignment, and ligament balancing will continue to evolve and will require a change in how we think about TKA. The 20\% dissatisfaction that Dr. Parratte mentioned, I believe, is in large part due to our lack of understanding the anatomy of each individual. We currently treat every 


\section{Clinical Faceoff}

knee the same when it comes to TKA, and while that may be good for $80 \%$, we leave another $20 \%$ dissatisfied. Newer imaging techniques and advanced technology should allow for customization of individual anatomy and may require a change in how we currently think about TKA.

Dr. Abdel: I would like to personally thank Dr. Springer and Dr. Parratte for their insight and expertise on this current controversy surrounding TKAs. While neither the gap-balancing technique nor measured resection technique is definitively better than the other, it is important to highlight that the key elements of both techniques are adequate placement of the components in the coronal, sagittal, and axial planes, as well as appropriate softtissue balancing. In reality, as noted by the invited experts, most arthroplasty surgeons utilize a hybrid approach that combines the two techniques.

Acknowledgments The author would like to thank Dr. Mark P. Abdel for his expertise and review of the manuscript.

\section{References}

1. Babazdeh S, Dowsey MM, Stoney JD, Choong PF. Gap balancing sacrifices joint-line maintenance to improve gap symmetry: A randomized controlled trial comparing gap balancing and measured resection. $J$ Arthroplasty. 2013.2.

2. Becker R, Malzdorf M, Starke C, Randolf P, Lohmann C. No difference between tibia-first and femurfirst techniques in TKA using computer-assisted surgery. Knee Surg Sports Traumatol Arthrosc. 2012;20: 2011-2016.

3. Dennis DA, Komistek RD, Kim RH, Sharma A. Gap balancing versus measured resection technique for total knee arthroplasty. Clin Orthop Relat Res. 2010;468:102-107.

4. Fehring TK. Rotational malalignment of the femoral component in total knee arthroplasty. Clin Orthop Relat Res. 2000:72-79.

5. Hanada H, Whiteside LA, Steiger J, Dyer P, Naito M. Bone landmarks are more reliable than tensioned gaps in TKA component alignment. Clin Orthop Relat Res. 2007;462: 137-142.

6. Kurtz S, Ong K, Lau E, Mowat F, Halpern M. Projections of primary and revision hip and knee arthroplasty in the United States from 2005 to 2030. J Bone Joint Surg Am. 2007;89:780-785.

7. Lee DH, Park JH, Song DI, Padhy D, Jeong WK, Han SB. Accuracy of soft tissue balancing in TKA: comparison between navigation-assisted gap balancing and conventional measured resection. Knee Surg Sports Traumatol Arthrosc. 2010;18: 381-387.

8. Matsumoto T, Muratsu H, Kubo S, Kuroda R, Kurosaka M. Intra-operative joint gap kinematics in unicompartmental knee arthroplasty. Clin Biomech (Bristol, Avon). 2013; 28:29-33.

9. Matsumoto T, Muratsu H, Kubo S, Matsushita $\mathrm{T}$, Ishida $\mathrm{K}$, Sasaki $\mathrm{H}$, Oka S, Kurosaka M, Kuroda R. Soft tissue balance using the tibia first gap technique with navigation system in cruciate-retaining total knee arthroplasty. Int Orthop. 2012;36: 975-980.

10. Nagai K, Muratsu H, Matsumoto T, Miya H, Kuroda R, Kurosaka M. Soft Tissue Balance Changes Depending on Joint Distraction Force in Total Knee Arthroplasty. $J$ Arthroplasty. 2013.

11. Parratte $S$, Blanc G, Boussemart $T$, Ollivier M, Le Corroller T, Argenson JN. Rotation in total knee arthroplasty: no difference between patient-specific and conventional instrumentation. Knee Surg Sports Traumatol Arthrosc. 2013;21:22132219.

12. Yoon JR, Jeong HI, Oh KJ, Yang JH. In vivo gap analysis in various knee flexion angles during navigation-assisted total knee arthroplasty. J Arthroplasty. 2013;28: 1796-1800. 